\title{
Materials for Drug Delivery: Innovative Solutions to Address Complex Biological Hurdles
}

\author{
Samir Mitragotri* and Joerg Lahann*
}

\section{Introduction}

$T_{\text {h }}$ the development of technologies to deliver biomolecules to the site of the disease so as to maximize therapeutic benefits, minimize side effects and enhance patient compliance. The key hurdle in this journey is the complexity of the path through which the drug has to navigate before arriving at the target site. Another hurdle is the limited availability of tools to control this navigation. Free drugs, when administered into bloodstream, are subjected to various metabolic processes, primarily renal clearance and distribution in non-target tissues. These processes not only reduce the drug concentration at the active target site but also increase the likelihood of unwanted side effects. The best examples of drugs suffering from these limitations are chemotherapeutic agents. While many chemotherapeutics are highly effective in a petri dish in vitro, clinical utility of these agents is often limited by severe restrictions on doses that are posed by their toxic side effects.

\author{
Prof. S. Mitragotri \\ Department of Chemical Engineering \\ University of California \\ Santa Barbara, Santa Barbara, CA 93106, USA \\ E-mail: samir@engineering.ucsb.edu \\ Prof. J. Lahann \\ Departments of Chemical Engineering \\ and Material Science and Engineering, \\ and Biomedical Engineering \\ Biointerfaces Institute \\ University of Michigan \\ Ann Arbor, MI 48105, USA \\ E-mail: lahann@umich.edu \\ Prof. J. Lahann \\ Institute for Functional Interfaces \\ Karlsruhe Institute of Technology \\ Karlsruhe, Germany, 76344
}

DOI: 10.1002/adma.201202080
These limitations can be potentially overcome by designing carriers that perform multiple tasks including encapsulation and controlled release, minimization of immune-clearance, penetration of biological barriers and targeting the disease site. ${ }^{[1-3]}$ This issue of Advanced Materials is dedicated to an overview of these challenges and innovative materials that are being developed to address them.

\section{Encapsulation and Release}

The central premise of encapsulation of drugs in carriers is to alter their biodistribution or deliver combinations of drugs through single administration. Carriers can protect drugs from the physiological clearance mechanisms and thus enhance stability, while allowing for defined release profiles to be governed by designed chemistries, rather than biological mechanisms. Carrier systems have been designed to exhibit extended release of therapeutic drug doses at the target site, while decreasing overall systemic drug dose to levels below the toxicity threshold. Benefits of this strategy include higher therapeutic efficacy, fewer side effects, and reduced number of administrations. The performance of drug carriers is strongly related to a number of key design parameters including the choice of material, carrier architecture and their surface modification.

\subsection{Choice of Carrier Material}

P olymers are one of the most commonly used materials for designing drug delivery carriers. ${ }^{[4]}$ The carrier material needs to be compatible with the drug to ensure stability during synthesis, storage, and administration. A number of different strategies have been employed to control the release profiles of polymeric delivery systems:

\section{Diffusion-based Release Systems}

In this configuration, drugs are loaded into an inert polymeric matrix and the drug release profile is governed by the diffusion of the drug through the matrix structure. This principle has been successfully employed in drug-eluting stents, ${ }^{[5]}$ and is also pursued in a number of particle-based delivery systems. ${ }^{[6]}$

\section{Solvent-Activated Release Systems}

Small molecule drugs and macromolecular drugs alike have been entrapped in water-swellable polymers, such as hydrogels. ${ }^{[6]}$ Upon exposure to a physiological environment, the hydrogel hydrates, expands, and releases the incorporated payload.

\section{Chemically Controlled Release Systems}

If the drug carrier is made of a material that is hydrolytically labile, the exposure to a physiological environment can trigger degradation of the polymer matrix. As the polymer degrades, previously incorporated payloads are released exhibiting profiles that can be engineered through modulation of the hydrolytic stability of the carrier polymers. ${ }^{[7]}$

\section{Responsive Release Systems}

Recent research has increasingly focused on the development of stimulusresponsive release systems. Specifically, biologically responsive nanoparticles have been at the forefront of research (see review by Grinstaff in this issue). The drug is encapsulated within a carrier particle that continues to shield the 
drug from the human body, even after administration. It is only after exposure to a specific stimulus, that the particles undergo a phase transition or chemical reaction that leads to the local release of the payload at a target site. Stimuliresponsive systems can respond to internal or external stimuli. Internal stimuli include changes in the $\mathrm{pH}$ value, temperature, or the presence of specific enzymes. External stimuli include exposure to light, electromagnetic waves, magnetic fields, or ultrasound. ${ }^{[7]}$ An elegant example is provided in the form of plasmonic nanobubbles for delivery of chemotherapeutics (see paper by Lapatko and co-workers in this issue).

\subsection{Carrier Architecture}

$W_{\text {hile diffusion-based and solvent- }}$ activated delivery systems often use isotropic-monolithic delivery architectures, particle engineers have increasingly sought after strategies that take advantage of finer control of the carrier architectures. ${ }^{[8]}$ For instance, in case of polymer micelles, the drug-containing core is encapsulated by a polymer shell that ensures particle stability, provides opportunities for surface modification, and controls the release profile of the drug. ${ }^{[9]}$ Thus, the circulation times and release profiles obtainable with polymerbased micelles can substantially diverge from conventional micelles. The exploration of increasingly complex carrier architectures becomes even more important since multifunctional particles are under development for combined imaging and therapeutic applications (e.g., theranostics). In addition, combination therapies may require the release of multiple therapeutics, ideally with decoupled release kinetics. See an article by Goodwill et al. in this issue for use of use of nanoparticles for imaging.

\section{Core-Shell Particles}

W hile there are a number of different particle types available for drug delivery, micelles are by the far most widely used delivery systems that have been developed in a number of variations. Different micelle types, such as worm-like micelles, or silica-cored micelles can show significant differences with respect to stability, efficacy, and circulation times. ${ }^{[10]}$ Among the most widely used synthetic systems are polymer micelles, circulating capsules, and nanocontainers. Complex release kinetics can be achieved with hierarchically structured micelle particles, such as vesosomes. ${ }^{[11]}$ In addition, peptide-based micelles have increasingly attracted a broad interest for biomedical applications. In this special issue, Tirrell and co-workers report peptide-based micelles that display a cytotoxic T-cell epitope for promotion of a protective immune response.

\section{Polymer-Drug Conjugates}

n polymer-drug conjugates, the drugs are directly bound to a carrier particle, which typically has a size of less than $10 \mathrm{~nm}$. Compared to the free drug, the carrier-bound drugs exhibit altered biodistribution, longer circulation times, and display in some cases a biologically responsive release trigger mechanism. ${ }^{[12]}$ Widely used carrier systems include hydrophilic polymers, dendrimers, albumin, and noble metal nanoparticles. ${ }^{[13]}$ An example that uses polymerdrug nanoparticles for two-stage release is provided by the so-called nanocells. ${ }^{[14]}$

\section{Compartmentalized Particles}

combination therapy often requires the release of two or more drugs with distinct therapeutic windows. Therefore, it would be beneficial, at least in some cases, to be able to deliver two different drugs with fully decoupled release profiles. At the first glance, the simplest solution would be the delivery of combinations of particles that carry different drugs. However, it cannot be ensured that the fate of two carriers in the body will be the same and that both particles will act upon a target cell at the same point in time. The solution to this fundamental dilemma may come from a slate of new carrier particles, so called multicompartmental particles, ${ }^{[15]}$ where a single particle is comprised of different nanocontainers, or compartments, each being made up of distinct drug/polymer combinations. ${ }^{[16]}$ In principle, it is even possible to design different release mechanisms into the same particle (see paper by Misra et al., this issue), or incorporate selective trigger mechanisms. This type of multicompartmental particles is also ideally suited for combined imaging and delivery applications (theranostics).

\section{Composite and Hybrid Particles}

$A$ highly sophisticated example of nanoemulsion composite microgels is presented by An et al. in this issue. In addition, there has been an increasing interest in organic/inorganic hybrid particles that feature dual functionality for imaging and therapy. Recent trends in this area have been reviewed in the paper by Harry and Sailor in this issue.

\subsection{Surface Modification}

Indepe ent the base polymers and the drug carrier architectures, there is almost always a need for surface modification of the carrier particles. With few exceptions, therapeutic nanoparticles injected in the body are cleared within minutes. ${ }^{[17-19]}$ For intravenous injection of particles, the upper size limit for effective drug delivery is determined by the capillary diameter. Micron-sized particles have been shown to have different velocities, diffusion characteristics and adhesion properties depending on the size. ${ }^{[20]}$ Particles less than $200 \mathrm{~nm}$ show improved circulation half-life compared to larger particles. However, unmodified nanoparticles often accumulate in liver and spleen within minutes to hours after intravenous injection. ${ }^{[21]}$ This defeats the purpose of encapsulation, since the drug is now released in these organs. In spite of major efforts during the last decades, clinical progress towards keeping carriers away from the liver and forcing them to accumulate in target tissues has been only moderately successful; certainly not living up to its potential to provide a "silver-bullet" for targeted drug delivery. 
The main hurdle in overcoming targeting challenge is the multitude of biological clearance mechanisms that exist in the human body including the reticuloendothelial system (RES). From administration to release - typically in the cytosol of a cell - a series of orthogonal clearance mechanisms prevent effective and targeted delivery. A typical target tissue in the body, for example tumor, is much smaller compared to the liver, lungs and spleen. Further, organs such as liver are highly vascularized. Collectively, the contact time of carriers with the RES is much higher than that with the tumor. This, in combination with the fact that RES macrophages are highly effective in capturing particles, far more than tumor cells, leads to much higher accumulation of particles in the liver and spleen compared to the tumor. To address these hurdles, current strategies seek to combine particle targeting via specific recognition of cancer cell biomarkers and decreased clearance due to "stealth" surface chemistries. A myriad of strategies have been developed to address these challenges; one at the time. For example, surface modification with polyethylene glycol (PEG) is commonly used to reduce RES clearance ${ }^{[22]}$ and tumor-targeting peptides to reduce off-site accumulation and cell-penetrating peptides to enhance cellular internalization (see a review by Ruoslahti in this issue). Incorporation of vastly different features into single particle architecture has been very difficult, if not impossible. In other words, enabling drug carriers to bypass a cascade of clearance mechanisms requires equipping the same particle with mutually exclusive sets of properties. A Multiple-Particles-in-One strategy, in which different parts of the same particle can be used to address the diverse set of functional requirements, can offer a solution to this problem (in analogy to a Swiss army knife). However, technical realization of such multifunctional particles has been an elusive challenge so far. The optimum surface density of binding sites vs. stealth areas will be one of the critical parameters, which will require entire novel particle architectures with defined surface patterns (i.e., activity clusters).
To extend the circulation time of drug delivery particles, the surface of the particles is typically covered by a layer of hydrogels. The most prominent examples include hydrophilic polymers, such as PEG, polysaccharides, such as dextran, or proteins, such as albumin, to minimize interactions of the particle with the immune system ${ }^{[23]}$ by decreasing particle hydrophobicity and surface charge density using hydrophilic polymers such as PEG ${ }^{[24-26]}$ and its variations. ${ }^{[27,28]}$ This has led to the generation of several methodologies to prepare "stealth" nanoparticles. PEG containing block copolymers such as poloxamines, poloxamers, PEG-PLGA have also been extensively studied. ${ }^{29-32]}$ Some of the liposome-PEG based delivery systems have also been shown to circulate for a couple of days. ${ }^{[3,34]}$ Particle size also plays a crucial role in determining the fate of the injected particles. ${ }^{[28,35,36]}$ Although these strategies have provided marked improvements over unmodified particles, they have several limitations. Nanoparticles are still removed from circulation possibly due to desorption of the surface coatings or activation of the complement.[37] Additionally, surface modified particles are rapidly recognized and removed from circulation upon repeated injection. ${ }^{[37-39]}$ Further, modification of nanomaterial surface with PEG compromises the ability to include targeting moieties on the particle surface.

In the past few years, there has been a paradigm shift in developing ways to fight the immune clearance. The new strategies take an "active" approach towards evading the immune system. Unlike the conventional approach of using hydrophilic polymers to minimize the interactions with the immune system, the new strategies aim at mastering "communication" with the immune system. For example, researchers have begun exploring the use of CD47, a ubiquitous self-marker as a means of disguising exogenous particles. ${ }^{[40]}$ Other innovative approaches such as engineering particle shape ${ }^{[41,42]}$ and mechanical properties $^{[18,43]}$ have also been developed. Particles with certain shapes have been shown to exhibit reduced macrophage uptake and enhanced circulation times and higher targeting to the diseased tissue. ${ }^{[36]}$ Flexibility of nanoparticles has also been shown to dramatically enhance circulation times and targeted accumulation. ${ }^{[18,43]}$ Nanoparticles have also been shown to hitchhike on red blood cells ${ }^{[44]}$ which leads to enhanced circulation. In another approach, nanoparticles have been encapsulated in autologous red blood cells to prolong the circulation. ${ }^{[45]}$ Cloaking nanoparticle surface with red blood cell membrane has also been shown to prolong their circulation. ${ }^{[46]}$ Additional strategies including glycocalyx-mimicking particles ${ }^{[47]}$ and lipoprotein-mimicking mimicking particles ${ }^{[48]}$ have also been explored. In yet another approach, researchers have developed strategies to optimize surface concentrations of PEG and targeting ligands to strike a balance between prolonged circulation and effective tissue accumulation. ${ }^{[49]}$ In another example, filomicelles, worm-like polymeric micelles, have been shown to circulate in mouse blood for around a week. ${ }^{[18]}$ In this issue, Discher et al. report the simulation studies on release of chemotherapeutic drugs from filomicelles.

\section{Penetration of Biological Barriers}

\subsection{Transport across Skin and Mucosal Barriers}

D ug carriers have to penetrate a variety of tissue- and cell-level barriers including skin and intestinal epithelium, mucosal tissues, endothelium, interstitium and cell membranes depending on the port of administration and ultimate target. Diffusion of nanoparticles across these tissues is very slow due to their size, especially across tissues such as skin and mucosa. Penetration of nanoparticles into skin is limited by its topmost, keratinized layer, the stratum corneum. Penetration into skin can be enhanced by treatment with permeabilizing agents such as chemicals, ${ }^{[50]}$ ultrasound, ${ }^{[51]}$ and microneedles. ${ }^{[52]}$ Several reports have documents the ability of soft nanoparticles such as liposomes, micelles and emulsions to enhance penetration into skin. ${ }^{[53]}$ 
Penetration of nano- and microparticles across the intestinal epithelium has also been extensively studied. ${ }^{[5,55]}$ This transport is limited by two barriers. The first barrier comprises the mucosal layer. Mucus plays an important role of trapping and clearance of particulates. Mucus forms polyvalent interactions with nanoparticles thus leading to their trapping and clearance. In order to penetrate mucus, synthetic nanoparticles must avoid adhesion to mucin fibers and be small enough to avoid significant steric inhibition by the dense fiber mesh. Nanoparticles as large as $500 \mathrm{~nm}$ have been shown to rapidly diffuse across human mucus after coating with polyethylene glycol. ${ }^{[56]}$ In this issue, Hanes et al. provide an overview of mucuspenetrating particles. In another recent study, the dependence of various particle parameters including size, shape and surface chemistry on mucociliary response was studied ex vivo. ${ }^{[57]}$ No significant differences in mucociliary clearance were found between particles of different size and shapes. The second barrier comprises the monolayer of the epithelial cells whose tight junctions offer a particularly strong barrier for entry of nanoparticles. Nanoparticles comprising various materials have been synthesized and used to deliver macromolecules, particularly insulin and vaccines. Pathways of nanoparticle transport have also been studied. ${ }^{[58]}$

\subsection{Transport in the Interstitium}

$T$ issue interstitium is a highly heterogeneous and structurally complex medium through which nanoparticles have to diffuse in order to reach the target cell membranes. ${ }^{[59]}$ The tumor interstitial matrix consists of a highly interconnected network of collagen fibers that interact with other molecules, such as proteoglycans and glycosaminoglycans. ${ }^{[60,61]}$ Transport of nanoparticles into tumors is further limited by the elevated interstitial pressure within the tumor. The primary mechanism of nanoparticle transport into interstitium is thus, diffusion. Diffusion of nanoparticles within the tumor depends on their size, charge and configuration as well as the physicochemical properties of the interstitial matrix. ${ }^{[62]}$ Small molecules diffuse fairly rapidly in the tumor interstitial matrix. However, the diffusion coefficient of nanoparticles and liposomes is dramatically reduced by high viscosity, low porosity and matrix interactions. Accessible volumes of tumors to macromolecular entities have been shown to range from 0.04 to 0.17. ${ }^{[63]}$ Collagenase treatment increases the porosity, which may in turn increase nanoparticle penetration. ${ }^{[64]}$ Indeed, the extravascular space available for large therapeutic agents decreases with the size of the agents.

The collagen matrix of the tumor poses a significant hurdle in nanoparticle diffusion. ${ }^{[65]}$ In the absence of sufficient diffusion through the interstitium, particles that extravasate from blood vessels may just localize on the periphery of the tumor. Particle charge may also play a significant role. Collagen fibers carry a slightly positive charge at neutral $\mathrm{pH}$, and hence may interact with negatively charged nanoparticles to form aggregates. ${ }^{[6]}$ In vitro studies using tumor spheroids, supplemented by mathematical models, have shown that nanoparticles of 20 and $40 \mathrm{~nm}$ in diameter are able to accumulate in the interior of the spheroid after treatment with collagenase, while $100 \mathrm{~nm}$ particles exhibited poor penetration in untreated spheroids with minor increase in penetration with collagenase treatment.

Several innovative strategies are being developed to enhance nanoparticle penetration into solid tissues. For example, peptides that exhibit enhanced tissue penetration have been identified. ${ }^{[67,68]}$ Peptides that possess a motif R/KXXR/K have been identified to bind to neuropilin-1 (NRP-1), a co-receptor for VEGF and lead to enhanced cellular entry. ${ }^{[69]}$ One prominent example of such peptides is iRGD (CRGDKGPD), which has been shown to deliver nanoparticles into tumors. ${ }^{[70]}$

Nanoparticle shape has also been shown to enhance interstitial transport. In a recent study, rod-shaped nanoparticles have shown enhanced diffusion in tumor interstitium compared to spherical particles. ${ }^{[71]}$ In another example, a multistage approach has been proposed where nanoparticles change their size to facilitate transport through interstitium. ${ }^{\text {[2] }}$ In this approach, the original 100-nm nanoparticles extravasate from the leaky regions of the tumor vasculature and shrink to $10 \mathrm{~nm}$, thus facilitating their penetration into the tumor parenchyma. Deep penetration into target tissues remains a significant hurdle. While a significant focus has been given to penetration into tumors, several other targets, in particular, brain suffer from the same limitation. Accordingly, new materials that are able to enhance penetration of nanoparticles into solid tissues are required.

\subsection{Internalization and Intracellular Trafficking within Target Cells}

Internalization of particles by cells is a complex process. There are some established thumb rules with respect to particle internalization into cells; particles $>1 \mu \mathrm{m}$ are internalized by phagocytosis and those between diameters of $0.2 \mu \mathrm{m}$ and $1 \mu \mathrm{m}$ are internalized by endocytosis. However, recent findings suggest that particles as large as $5 \mu \mathrm{m}$ can be endocytosed through receptor mediated endocytosis. ${ }^{[73,74]}$ The extent of internalization of particles depends on various parameters including surface chemistry, size and shape. ${ }^{[75]}$ Among these, surface chemistry is the most studied parameter. Association of carriers with cell membranes depends extensively on surface hydrophobicity and charge. Hydrophobic particles are known to better associate with cell membranes, although such association is non-specific. The same is also true about surface charge. Positively charged particles have a high affinity with negatively charged cell membranes. In spite of their strong association with cell membranes, hydrophobic and positively charged particles are not actively used due to their non-specific association with cells.

Shape of nanoparticles has also been engineered to enhance intracellular drug delivery. Specifically, needleshaped particles have been shown to enhance intracellular delivery of siRNA compared to spherical particles due 
to shape-induced membrane permeabilization. ${ }^{[76,77]}$ In another study, rodshaped nanoparticles have been shown to exhibit enhanced intracellular uptake compared to spherical particles. ${ }^{[74]}$ Another study has shown that particle size and shape play an intriguing role in endothelial targeting, receptor mediated endocytic internalization and intracellular trafficking. ${ }^{[36]}$ Particle geometry plays an important role in therapeutic delivery to the endothelium. Surface architecture has also been shown to play a significant role in nanoparticle uptake. Studies from the Stellacci group have shown that striated nanoparticles exhibit membrane penetration and delivery of materials in the cytoplasm ${ }^{[78]}$ (also, see a paper by Stellacci et al. in this issue).

\section{Accumulation at the Target}

$T_{\text {are }}$ remains one of the key challenges in drug delivery. Two key principles are followed for targeting nanoparticles to tissues; passive targeting that relies on leaky vasculature and active targeting that uses the presence of specific ligands.

\subsection{Passive Targeting: Enhanced Permeation and Retention}

$T$ umor vasculature exhibits less organized structure and higher permeability compared to vasculature in healthy tissues. ${ }^{[79,80]}$ Characteristics of the EPR effect include extensive angiogenesis, defective vascular architecture and impaired lymphatic drainage/recovery system of the tumors. Solid and rapidly growing tumors possess inadequate supply of nutrients and oxygen and hence possess extensive angiogenesis, resulting in a high vascular density. Under such conditions, blood vessels form in an unorganized manner and possess defective architecture of the vascular endothelium with large gaps in endothelium cell-cell junctions thus leading to high permeability. The EPR effect provides an opportunity for more selective targeting of nanoparticles to tumors.
The ability of nanoparticles to exhibit EPR depends on several parameters including size and surface charge. As the primary factor, the ability of nanoparticles to accumulate in tissues through EPR depends on the circulation time of nanoparticles. Most nanoparticles are rapidly cleared from circulation due to recognition by the RES. To take advantage of the EPR effect, it is critical for the nanocarriers to evade immune surveillance and circulate for a prolonged period. Particles smaller than $10 \mathrm{~nm}$ are filtered by the kidney whereas particles larger than 100-200 $\mathrm{nm}$ are captured by the liver. Therefore, particles within this range are likely to exhibit high EPR, ${ }^{[81]}$ although micron-size particles have also been shown to exhibit EPR. Surface charge also impacts the EPR effect. Specifically, positively charged particles are rapidly cleared from circulation, thus limiting their ability to accumulate in tumors. On the other hand, neutral and negatively charged particles are likely to exhibit longer circulation and higher tumor accumulation.

Particle shape is also known to impact the EPR effect. Worm-shaped flexible filomicelles have been shown to exhibit higher tumor accumulation due to EPR compared to their spherical counterpart, likely due to their longer circulation. ${ }^{[18]}$ In another study, experiments with discoidal particles showed that EPR-mediated accumulation of particles in tumors exhibited dependence on shape. Specifically, discoidal-shaped particles exhibited higher accumulation. ${ }^{[82]}$ Particles that change size have also been proposed to enhance the EPR effect. Specifically, nanoparticles that are able to increase their size in response to the low extracellular $\mathrm{pH}(6.4 \sim 6.8)$ in a tumor area may be physically confined in the tumor due to aggregation-induced increase in size (i.e., size increase over endothelial gap junction) upon extravasation. In vitro studies have reported hydrogel nanoparticles composed of pullulan acetate (PA)sulfadimethoxine (SDM) that exhibit remarkable size change upon exposure to tumoral $\mathrm{pH}$ ( $\mathrm{pH} 6.8$ ), resulting from hydrophobic transition of SDM. ${ }^{[83]}$ In some cases, it is beneficial to tune rather than avoid immunity. The progress report by Irvine and collaborators in this issue describes novel routes to engineer synthetic particles with designable immunity.

\subsection{Active Targeting}

$T$ argeted delivery is achieved by surface functionalization of particles with biomolecules such as peptides, ${ }^{[84]}$ aptamers, ${ }^{[85]}$ and antibodies. ${ }^{[86]}$ A number of tumor-specific antibodies and drugs targeting specific proteins and small molecules are undergoing both preclinical and clinical trials. Researchers have also developed strategies to optimize surface concentrations of PEG and targeting ligands to strike a balance between prolonged circulation and effective tissue accumulation. These include optimization of randomly distributed PEG and aptamers on nanoparticle surface ${ }^{[31]}$ and fabrication of compartmentalized particles. ${ }^{[87,88]}$ Kokkoli et al. provide a review of peptide-targeted lipid nanoparticles for anti-cancer drug delivery in this issue.

Particle shape also affects the targeting efficiency. Using theoretical models to supplement experimental data, the effect of shape on the transport of cells and carriers through the blood vessels has been studied. ${ }^{[89]}$ Margination, firm adhesion and internalization are important features of transport of carriers through capillaries. Particle interaction with the vasculature includes receptor-ligand interactions and non-specific interactions such as van der Waals, electrostatic and steric interactions. A neutrally buoyant spherical particle moving in proximity to a wall can drift laterally only in presence of an external force whereas non-spherical particles exhibit more complex motions with tumbling and rolling which can be exploited to control their margination dynamics without any need for lateral external forces. For non-spherical particles, it has been shown that the lateral drifting velocity is directly related to their aspect ratio, with a maximum between the two extremes: sphere, with aspect ratio unity, and disk, with aspect ratio infinity. Discoidal particles have been shown to marginate more than quasi-hemispherical which in turn 
marginated more than spherical particles in a gravitational field. ${ }^{[9]}$ Such models provide profound fundamental understanding and predictive capabilities for carrier behavior in flow and will prove to be instrumental in efficient design of carriers.

Strategies to improve targeting have typically made use of discovery and utilization of new chemical ligands. However, recent studies show that other physical factors such as size and shape impact the ability of particles to target. In this issue, Doshi et al. describe the ability of platelet-mimicking particles to target, in vitro, surfaces that mimic damaged endothelium. Development of synthetic materials with the complex biochemical and physical attributes of natural cells may open new opportunities in medical therapy. Reports have been published on synthetic blood cells ${ }^{[91,92]}$ and RGDcoated nanoparticles reproducing some of the functions exhibited by platelets during aggregation. ${ }^{\text {[9] }] ~ I n ~ g e n e r a l, ~ b i o-~}$ logical objects such as viruses, bacteria, and cells provide a motivating paradigm for designing drug carriers. This issue includes a review by Little et al. on this topic.

\section{Summary}

Recent literature including the publications in this issue demonstrates the potential of advanced materials in addressing unmet needs in drug delivery. A variety of discoveries and innovations have helped the design and fabrication of novel drug carrier systems. Collectively, these innovations are making a strong progress towards addressing the challenges in the field of therapeutic delivery.

[1] S. Moghimi, FASEB J. 2005, 19, 311-330.

[2] N. Doshi, S. Mitragotri, Adv. Funct. Mater. 2009, 19, 3843-3854.

[3] O. Farokhzad, R. Langer, Adv. Drug Delivery Rev. 2006, 58, 1456-1459.

[4] R. Langer, N. A. Peppas, AiChE J. 2003, 49, 2990-3006.

[5] S. Commandeur, H. M. van Beusekom, W. J. van der Giessen, J. Interv. Cardiol. 2006, 19, 500-6.
[6] R. S. Langer, N. A. Peppas, Biomaterials 1981, 2, 201-214.

[7] S. Kim, J. H. Kim, O. Jeon, I. C. Kwon, K. Park, Eur. J. Pharm. Biopharm. 2009, 71, 420-30.

[8] J. W. Yoo, D. J. Irvine, D. E. Discher, S. Mitragotri, Nat. Rev. Drug Discov. 2011, 10, 521-35.

[9] C. E. Ashley, E. C. Carnes, G. K. Phillips, D. Padilla, P. N. Durfee, P. A. Brown, T. N. Hanna, J. Liu, B. Phillips, M. B. Carter, N. J. Carroll, X. Jiang, D. R. Dunphy, C. L. Willman, D. N. Petsev, D. G. Evans, A. N. Parikh, B. Chackerian, W. Wharton, D. S. Peabody, C. J. Brinker, Nat. Mater. 2011, 10, 389-97.

[10] D. J. Irvine, Nat. Mater. 2011, 10, 342-3.

[11] E. T. Kisak, B. Coldren, C. A. Evans, C. Boyer, J. A. Zasadzinski, Curr. Med. Chem. 2004, 11, 199-219.

[12] E. S. Lee, K. Na, Y. H. Bae, J. Control. Release 2005, 103, 405-18.

[13] R. Duncan, Nat. Rev. Drug Discov. 2003, 2, 347-60.

[14] S. Sengupta, D. Eavarone, I. Capila, G. Zhao, N. Watson, T. Kiziltepe, R. Sasisekharan, Nature 2005, 436, 568-72.

[15] J. Lahann, Small 2011, 7, 1149-56.

[16] K. J. Lee, S. Hwang, J. Yoon, S. Bhaskar, T.-H. Park, J. Lahann, Macromol. Rapid Commun. 2011, 32, 431-7.

[17] Z. Liu, X. Li, S. M. Tabakman, K. Jiang, S. Fan, H. Dai, Proc. Natl. Acad. Sci. USA 2008, 105, p. 1410

[18] Y. Geng, P. Dalhaimer, S. Cai, R. Tsai, M. Tewari, T. Minko, D. E. Discher, Nature 2007, 2, 249-255.

[19] A. Ayyagari, X. Zhang, K. B. Ghaghada, A. Annapragada, X. Hu, R. V. Bellamkonda, Magnetic Resonance Med. 2006, 55, 1023.

[20] V. R. Shinde Patil, C. J. Campbell, Y. H. Yun, S. M. Slack, D. J. Goetz, Biophys. J. 2001, 80, 1733-1743.

[21] V. P. Torchilin, AAPS J. 2007, 9, E128-47.

[22] S. M. Moghimi, A. C. Hunter, J. C. Murray, Pharmacol. Rev. 2001, 53, 283-318.

[23] J. W. Yoo, E. Chambers, S. Mitragotri, Curr. Pharm. Des. 2010, 16, 2298-2307.

[24] A. Vonarbourg, C. Passirani, P. Saulnier, J.-P. Benoit, Biomaterials 2006, 27 4356-4373.

[25] U. Wattendorf, H. Merkle, J. Pharmaceut. Sci. 2008, 97, 4655-4669.

[26] L. E. van Vlerken, T. K. Vyas, M. M. Amiji, Pharmaceut. Res. 2007, 24, 1405-1414.

[27] G. Prencipe, S. M. Tabakman, K. Welsher, Z. Liu, A. P. Goodwin, L. Zhang, J. Henry, H. J. Dai, J. Am. Chem. Soc. 2009, 131, 1360-1361.

[28] C. Fang, B. Shi, Y.-Y. Pei, M.-H. Hong, J. Wu, H.-Z. Chen, Eur. J. Pharmaceut. Sci. 2006, 27, 27-36.
[29] J. Cheng, B. A. Teply, I. Sherifi, J. Sung, G. Luther, F. X. Gu, E. Levy-Nissenbaum, A. F. Radovic-Moreno, R. Langer, O. C. Farokhz, Biomaterials 2007, 28, 869-876.

[30] Y. Sheng, J. Deng, X. Luo, X. Zhang, $X$. Zeng, M. Feng, S. Pang, Biomaterials 2009, 20, 1217-1233.

[31] F. Gu, L. Zhang, B. A. Teply, N. Mann, A. Wang, A. F. Radovic-Moreno, R. Langer, O. C. Farokhzad, Proc. Natl. Acad. Sci. USA 2008, 105, 2586.

[32] M. Neu, O. Germershaus, S. Mao, K. H. Voigt, M. Behe, T. Kissel, J. Controlled Release 2007, 124, 69-80.

[33] S. Moghimi, J. Szebeni, Progr. Lipid Res. 2003, 42, 463-478.

[34] T. Allen, Trends Pharmacol. Sci. 1994. 15, 215-220.

[35] J. A. Champion, A. Walker, S. Mitragotri, Pharmaceut. Res. 2008, 25, 1815-1821.

[36] S. Muro, C. Garnacho, J. A. Champion, J. Leferovich, C. Gajewski, E. H. Schuchman, S. Mitragotri, V. R. Muzykantov, Mol. Ther. 2008, 16, 1450-8.

[37] S. M. Moghimi, A. C. Hunter, Critical Rev. Therapeut. Drug Carrier Syst. 2001, 18, 527-550.

[38] T. I. Armstrong, S. M. Moghimi, S. S. Davis, L. Illum, Pharmaceut. Res. 1997, 14, 1629-1633.

[39] S. M. Moghimi, T. Gray, Clin. Sci. 1997, 93, 371-379.

[40] P.-A. Oldenborg, A. Zheleznyak, Y.-F. Fang, C. F. Lagenaur, H. D. Gresham, F. P. Lindberg, Science 2000, 288, 2051-2054

[41] J. Champion, Y. Katare, S. Mitragotri, Proc. Natl. Acad. Sci. USA 2007, 104, 11901.

[42] D. Dendukuri, D. C. Pregibon, J. Collins, T. A. Hatton, P. S. Doyle, Nat. Mater. 2006, 5, 365-369.

[43] K. A. Beningo, Y. Wang, J. Cell Sci. 2002, 175, 849-856.

[44] E. Chambers, S. Mitragotri, Exp. Biol. Med. 2007, 232, 958

[45] A. Antonelli, C. Sfara, L. Mosca, E. Manuali, M. Magnani, J. Nanosci. Nanotechnol. 2008, 8, 2270-8.

[46] C. M. Hu, L. Zhang, S. Aryal, S. C. Cheung, R. H. Fang, L. Zhang, Proc. Natl. Acad. Sci. USA 2011, 108, 10980-5.

[47] J. Zhu, J. Xue, Z. Guo, L. Zhang, R. E. Marchant, Bioconjug. Chem. 2007, 18(5), 1366-9.

[48] S. Utreja, A. J. Khopade, N. K. Jain, Acta Helv. 1999, 73, 275-9.

[49] F. Gu, L. Zhang, B. A. Teply, N. Mann, A. Wang, A. F. Radovic-Moreno, R. Langer, O. C. Farokhzad, Proc. Natl. Acad. Sci. USA 2008, 105, 2586-91.

[50] P. Karande, A. Jain, S. Mitragotri, Nat. Biotechnol. 2004, 22, 192-197. 
[51] M. Ogura, S. Paliwal, S. Mitragotri, Adv. Drug Delivery Rev. 2008, 60, 1218-23.

[52] M. R. Prausnitz, J. A. Mikszta, M. Cormier, A. K. Andrianov, Curr. Top. Microbiol. Immunol. 2009, 333, 369-93.

[53] G. Cevc, U. Vierl, J. Control. Release 2010, 141, 277-99.

[54] G. P. Carino, E. Mathiowitz, Adv. Drug Delivery Rev. 1999, 35, 249-257.

[55] S. Sakuma, S. Sakuma, R. Sudo, N. Suzuki, H. Kikuchi, M. Akashi, M. Hayashi, J. Control. Release 2002, 81, 281-90.

[56] S. K. Lai, Y.-Y. Wang, K. Hidac, R. Cone, J. Hanes, Proc. Natl. Acad. Sci. USA 2007, 104, 1482-7.

[57] J. Kirch, M. Guenther, N. Doshi, U. F. Schaefer, M. Schneider, S. Mitragotri, C. M. Lehr, J. Control. Release 2012, 159, 128_134.

[58] X. Xie, Q. Tao, Y. Zou, F. Zhang, M. Guo, Y. Wang, H. Wang, Q. Zhou, S. Yu, J. Agric. Food. Chem. 2011, 59, 9280-9.

[59] R. K. Jain, T. Stylianopoulos, Nat. Rev. Clin. Oncol. 2010, 7(11), 653-64.

[60] H. F. Dvorak, N. Engl. J. Med. 1986, 315, 1650-9.

[61] L. Ronnov-Jessen, O. W. Petersen, M. J. Bissell, Physiol. Rev. 1996, 76, 69-125.

[62] R. K. Jain, Cancer Res. 1987, 47, 3039-51.

[63] A. Krol, J. Maresca, M. W. Dewhirst, F. Yuan, Cancer Res. 1999, 59, 4136-41.

[64] T. T. Goodman, J. Chen, K. Matveev, S. Pun, Biotechnol. Bioeng. 2008, 101 388-99.

[65] A. Pluen, Y. Boucher, S. Ramanujan, T. D. McKee, T. Gohongi, E. di Tomaso, E. B. Brown, Y. Izumi, R. B. Campbell, D. A. Berk, R. K. Jain, Proc. Natl. Acad. Sci. USA 2001, 98, 4628-33.
[66] T. Stylianopoulos, M.-Z. Poh, N. Insin, M. G. Bawendi, D. Fukumura, L. L. Munn, R. K. Jain, Biophys. J. 2010, 99, 1342-9.

[67] T. Hsu, S. Mitragotri, Proc. Natl. Acad. Sci. USA 2011, 108, 15816-21.

[68] P. B. Robbins, S. F. Oliver, S. M. Sheu, J. B. Goodnough, P. Wender, P. A. Khavari, Biotechniques 2002, 33, 190-2, 194.

[69] T. Teesalu, K. N. Sugahara, V. R. Kotamraju, E. Ruoslahti, Proc. Natl. Acad. Sci. USA 2009, 106, 16157-62.

[70] K. N. Sugahara, T. Teesalu, P. P. Karmali, V. R. Kotamraju, L. Agemy, D. R. Greenwald, E. Ruoslahti, Science 2010, 328, 1031-5.

[71] V. P. Chauhan, V. P. Chauhan, Z. Popovic O. Chen, J. Cui, D. Fukumura, M. G. Bawendi, R. K. Jain, Angew. Chem. Int. Ed. Engl. 2011, 50, 11417-20.

[72] C. Wong, T. Stylianopoulos, J. Cui, J. Martin, V. P. Chauhan, W. Jiang, Z. Popovic, R. K. Jain, M. G. Bawendi, D. Fukumura, Proc. Natl. Acad. Sci. USA 2011, 108 2426-31.

[73] S. Muro, C. Garnacho, J. A. Champion, J. Leferovich, C. Gajewski, Molecular Therapy 2008, 16(8), 1450-1458.

[74] S. Gratton, P. A. Ropp, P. D. Pohlhaus, J. C. Luft, V. J. Madden, Mary E. Napier, M. DeSimone, Proc. Natl. Acad. Sci. USA 2008, 105, 11613.

[75] J. L. Perry, K. P. Herlihy, M. E. Napier, J. M. DeSimone, Acc. Chem. Res. 2011, 44, 990-998.

[76] N. Doshi, S. Mitragotri, J. R. Soc, Interface 2010, 7(Suppl 4), S403-S410.

[77] P. Kolhar, N. Doshi, S. Mitragotri, Small 2011, 7, 2094-100

[78] A. Verma, O. Uzun, Y. H. Hu, Y. Hu, H. S. Han, N. Watson, S. Chen, D. J. Irvine, F. Stellacci, Nat. Mater. 2008, 7, 588-95.
[79] H. Maeda, Bioconjug. Chem. 2010, 21, 797-802

[80] H. Maeda, Y. Matsumura, Adv. Drug Delivery Rev. 2011, 63, 129-30.

[81] S. Acharya, S. K. Sahoo, Adv. Drug Delivery Rev. 2011, 63, 170-83.

[82] G. Adriani, M. D. de Tullio, M. Ferrari, F. Hussain, G. Pascazio, X. Liu, P. Decuzzi, Biomaterials 2012, 33(22), 5504 13.

[83] K. Na, Y. H. Bae, Pharm. Res. 2002, 19 , 681-8.

[84] W. Arap, R. Pasqualini, E. Ruoslahti, Science 1998, 279, 377.

[85] O. Farokhzad, J. Cheng, B. A. Teply, I. Sherifi, S. Jon, P. W. Kantoff, J. P. Richie, R. Langer, Proc. Natl. Acad. Sci. USA 2006, 103, 6315-6320.

[86] J. Sudimack, R. Lee, Adv. Drug Delivery Rev. 2000. 41, 147-162.

[87] K. Roh, D. Martin, J. Lahann, Nat. Mater. 2005, 4, 759-763.

[88] K. H. Roh, D. C. Martin, J. Lahann, J. Am Chem. Soc. 2006, 128, 6796-7.

[89] P. Decuzzi, R. Pasqualini, W. Arap, M. Ferrari, Pharmaceut. Res. 2009, 26, 235243.

[90] F. Gentile, A. Curcio, C. Indolfi, M. Ferrari, P. Decuzzi, J. Biomech. 2008, 41, 2312-2318.

[91] N. Doshi, A. Zahr, S. Bhaskar, J. Lahann, S. Mitragotri, Proc. Natl. Acad. Sci. USA 2009, 106, 21495.

[92] T. Merkel, S. Jones, K. Herlihy, F. Kersey A. Shields, M. Napier, J. Luft, H. Wu H. Zamboni, Proc. Natl. Acad. Sci. USA 2011, 108, 586.

[93] J. P. Bertram, C. A. Williams, R. Robinson, S. S. Segal, N. T. Flynn, E. B. Lavik, Sci. Transl. Med. 2009, 1, 11 ra22. 